\title{
Instrumenteca RA: Um Objeto de Aprendizagem para Auxiliar a Percepção Musical através da Diferenciação dos Sons de Instrumentos Musicais
}

\section{Instrumenteca RA: A Learning Object to Assist Musical Perception through the Differentiation of Musical Instrument Sounds}

\author{
Daniel Yokoyama Fecchio - FCI - Universidade Presbiteriana Mackenzie - \\ daniel.fecchio@hotmail.com \\ Fernando José Germinio Fenley - FCI - Universidade Presbiteriana Mackenzie - \\ fefenley@gmail.com
}

Samara Soares Nunes- FCI - Universidade Presbiteriana Mackenzie -samaraplim@hotmail.com

Ana Grasielle Dionísio Corrêa - FCI/ Programa de Pós-Graduação em Distúrbios do Desenvolvimento - Universidade Presbiteriana Mackenzie - ana.correa@mackenzie.br

Valeria Farinazzo Martins - FCI/ Programa de Pós-Graduação em Distúrbios do Desenvolvimento - Universidade Presbiteriana Mackenzie - valeria.farinazzo@mackenzie.br

Resumo. A música é uma importante ferramenta no desenvolvimento das crianças, pois envolve percepção, sentimentos, experiências, criação e reflexão. Entretanto, a maioria das instituições de educação infantil não oferece práticas pedagógicas que explorem a linguagem da música, privando as crianças destas experiências. $O$ uso de objeto de aprendizagem musical pode proporcionar os conceitos básicos de percepção musical para as crianças, despertando assim o interesse em desenvolver as capacidades musicais. Este artigo apresenta um objeto de aprendizagem em percepção musical composto por duas aplicações de Realidade Aumentada (Instrumenteca RA e Quiz). Testes com 12 usuários foram realizados no intuito de verificar a satisfação dos usuários assim como o aprendizado na utilização das aplicações.

Palavras-Chave: aprendizado musical, realidade aumentada, percepção musical.

\begin{abstract}
Music is an important tool in the development of children since it involves perception, feelings, experiences, creation and reflection. However, most early childhood education institutions do not offer pedagogical practices that exploit the language of music, depriving children of these experiences. The use of musical learning objects provides the basic concepts of musical perception for children, arousing interest in developing musical abilities. This article presents an object of learning in musical perception composed by two applications of Augmented Reality (AR and Quiz Instrumenteca). Tests with 12 users were performed to verify the their satisfation and learning using the applications.
\end{abstract}

Keywords: musical learning, augmented reality, musical perception. 


\section{Introdução}

A música é reconhecida por muitos pesquisadores como uma espécie de modalidade que desenvolve a mente humana, promove o equilíbrio, proporcionando um estado agradável de bem-estar, facilitando a concentração e o desenvolvimento do raciocínio, além de ser uma maneira de expressar sentimentos, como afirma Rodrigues e Rosin (2011). A música está presente em todas as culturas e contribui na educação, inclusive nas áreas pedagógicas e sociais, como compreensão, tolerância e convivência, além de melhorar o raciocínio lógico e desenvolver a capacidade de expressão e criatividade (Souza, 2014). De acordo com Jesus et al. (2010), o ensino musical na educação infantil tem o objetivo de introduzir a diversidade musical para a criança, através de diversos sons e trabalhar elementos musicais como melodia, harmonia e ritmo.

Musicalização infantil, de acordo com Rodrigues (2016), é o desenvolvimento do senso musical das crianças, assim como a sensibilidade, expressão, ritmo e ouvido musical, incluindo a criança no mundo da música. O processo de musicalização tem o objetivo de fazer com que a criança se torne um ouvinte sensível de música, ampliando o seu universo sonoro.

Gordon (2000) complementa a definição de Rodrigues (2016) sobre musicalização infantil: a falta de compreensão dos elementos musicais faz com que as pessoas percebam apenas a letra, que é a parte menos musical. Quando a letra é omitida, é como se não existisse mais a música. Entretanto, a música existe independente da letra. Para perceber o que há por trás das letras, é necessário algum nível de vivência com os elementos musicais. Quanto melhor os alunos compreenderem a música, melhor conseguirão apreciá-la.

De acordo com a Associação Brasileira de Educação Musical (ABEM, 2016), o ensino musical pode ser trabalhado junto com as aulas de educação artística propondo novos projetos com um dos objetivos de desenvolver diversas habilidades sensóriomotoras nas crianças. $\mathrm{O}$ ensino das artes incorporados em projeto educacionais, segundo a Lei $\mathrm{n}^{\circ} 11.769$, vem ao encontro de propostas inovadoras, em que a expressão cultural e artística é reconhecida como dimensões insubstituíveis e, portanto, únicas no sentido de promover o desenvolvimento humano.

Por outro lado, a Realidade Aumentada (RA) tem sido apontada pela Horizon Report (Johnson, 2016) como uma das tecnologias que irão revolucionar a educação como um todo. Ela é a união de objetos virtuais com o ambiente físico, mostrada ao usuário, em tempo real, com o apoio de algum dispositivo tecnológico, usando a interface do ambiente real, adaptada para visualizar e manipular os objetos reais e virtuais. (Kirner; Kirner, 2008). Sua utilização para a educação apresenta grande potencial, especialmente com a motivação que pode resultar aos alunos possibilitando-os um acesso ao mundo virtual para interagir com objetos limitados à sua imaginação (Bower et al., 2014). Assim, a inserção de objetos virtuais aos objetos reais pode ampliar a possibilidade de informação disponibilizada com a ajuda de várias mídias concomitantes (som, imagem, vídeos, por exemplo), além de permitir um aumento de motivação dos alunos.

Este artigo tem como objetivo apresentar como o uso de Realidade Aumentada pode ser utilizado para o ensino musical, tornando o aprendizado mais motivador. $\mathrm{O}$ artigo apresenta um objeto de aprendizagem composto por dois aplicativos denominados Instrumenteca RA e Instrumenteca Quiz, que serão descritos na seção 4. Este objeto de apresendizagem procurou envolver os conceitos básicos provenientes da percepção musical e os tipos de instrumentos musicais. Foi realizado um estudo sobre a satisfação 
do usuário no uso de tais instrumentos com um grupo de crianças e também foi medido o aprendizado sobre o tema. Os resultados são apresentados neste trabalho.

$\mathrm{O}$ artigo está organizado conforme se segue. Na seção 2 são apresentados o referencial teórico fundamental para o entendimento do restante do artigo. A seção 3 traz os trabalhos relacionados. Na seção 4 está descrito o método da pesquisa. Na seção 5 são apresentados os resultados e discussões. Finalmente, na seção 6, estão a conclusão e os trabalhos futuros.

\section{Referencial Teórico}

Para que seja possível compreender o este projeto como um todo, faz-se necessário conhecer os conceitos envolvidos em seu desenvolvimento.

\subsection{Percepção Musical}

Segundo Bernardes (2001), a percepção musical é a capacidade de perceber e identificar as ondas sonoras como parte de uma linguagem musical. Envolve a identificação de sons em suas características como altura, timbre, afinação, ritmo, melodia, harmonia, intensidade, entre outras características. É uma habilidade essencial para o desenvolvimento e progresso contínuo, pois como a música é uma linguagem, é preciso aprender a falar, escrever e ouvir essa linguagem.

De acordo com Borges e Penna (2015), com o desenvolvimento da percepção musical é possível perceber os sons como padrões repletos de significados e, assim, ouvilos e executá-los. É preciso compreender o que é ouvido e esse resultado é obtido através do desenvolvimento da audição e execução.

\subsection{Diferenciação dos Instrumentos Musicais através do Timbre}

Um instrumento musical é um objeto, construído com o propósito de produzir sons. A classificação de instrumentos musicais consiste na divisão dos mesmos de acordo com suas características. Todos estes instrumentos são classificados, segundo Silva e Toni (1990), de duas maneiras, conforme a característica de seu som ou de que forma produzem o som:

- Cordofones: classificados como instrumentos de cordas, são aqueles instrumentos em que o som é obtido pela vibração das cordas. As cordas podem ser dedilhadas, percutidas ou colocadas em vibração com um arco (friccionadas). São exemplos deste tipo de características: baixo, guitarra, piano, violão, violino e violoncelo.

- Idiofones: classificados como instrumentos de percussão, são aqueles instrumentos que produzem som ao serem percutidos, provocando a vibração de todo o instrumento musical. São exemplos: maracas (maracá, bapo, maracaxá ou xuatê) e xilofone.

- Membranofones: classificados como instrumentos de percussão, são aqueles instrumentos em que o som é produzido quando são percutidos sobre uma membrana esticada que entra em vibração. As membranas podem ser de origem animal, sintéticas ou até mesmo de tecido. São exemplos: conga e djembê.

- Aerofones: classificados como instrumentos de sopro, são aqueles instrumentos que produzem som quando o ar ao ser neles introduzido entre em vibração, excitando os componentes do instrumento musical. São exemplos: flauta de pã e trompete. 


\subsection{Realidade Aumentada (RA)}

De acordo com Azuma (1997) e Billinghurst (2012), RA é uma tecnologia que permite ao usuário visualizar objetos virtuais sobrepostos ou compostos com o mundo real. Ao contrário da Realidade Virtual, que é totalmente artificial, RA une o mundo real e o mundo virtual, através da inserção de objetos virtuais em um ambiente real, sem ocultar do usuário o que está em sua volta em tempo de execução real e registrado em 3D. RA possibilita o manuseio de objetos virtuais com as mãos, enquanto a visualização pode ocorrer por meio de um cardboard, ou seja, um óculos de Realidade Virtual. Além disso, RA é intuitiva, natural e atrativa, o que dispensa ensinamentos prévios para sua utilização.

O funcionamento básico de RA se dá através de uma câmera e um software que fará a ligação entre eles. O software de RA é capaz de reconhecer marcadores feitos de papel, assim, utiliza técnicas de Visão Computacional. O reconhecimento é feito através da webcam que captura imagens do mundo real, faz a leitura do marcador e, a partir de seu reconhecimento, adiciona um elemento $3 \mathrm{D}$ na cena real, misturando os dois ambientes, real e virtual, proporcionando uma interação mais natural e intuitiva. No Instrumenteca RA é utilizado um óculos Google Cardboard que permite visualizar aplicações de RA em primeira pessoa, ou seja, o usuário olha para o marcador enquanto ele é transformado em um objeto 3D. O Google Cardboard é composto por duas lentes acopladas a um compartimento que se encaixa ao rosto do usuário, o smartphone é colocado dentro do compartimento dos óculos, pela qual é possível a visualização um ambiente de RA.

\section{Trabalhos Relacionados}

Foram encontrados na literatura vários trabalhos relacionados a objetos de aprendizagem para ensino de música. Berry et al (2003) apresentam o Music Table, um sistema de RA para composição musical. O usuário organiza cartões feitos de papel em uma superfície, e cada cartão representa uma nota musical. A posição dos cartões na mesa é controlada por uma câmara ligada a um computador conectado a um monitor de vídeo na qual as imagens capturadas pela câmera são exibidas para o usuário. O software gera imagens de criaturas animadas, fornecendo o feedback para o usuário. Trata-se, portanto, do ensino de música e não de percepção musical que precede este conceito.

Corrêa, Ficheman e Lopes (2012) apresentam um desenvolvimento de uma interface musical tangível com o uso de RA, juntando duas áreas de pesquisa, as interfaces tangíveis e a música. Como resultado, houve a criação de um jogo, onde os usuários interagem com cartões marcadores para apreciar o timbre do instrumento musical desejado através da interação com cubos virtuais coloridos. Trata-se, também, de ensino musical.

Corrêa et al. (2012) apresentam o jogo MusicandoRA, onde o objetivo principal é a interação de conteúdos musicais como instrumentos musicais, notas musicais e sons com as crianças. Através de uma webcam, os usuários interagem com cartões marcadores, assim eles visualizam instrumentos em diferentes perspectivas, escutam os timbres das notas musicais e podem jogar um jogo de memória musical para adivinhar os sons dos instrumentos. Este trabalho está alinhado a este projeto, pois trata do ensino de percepção musical.

Gomez, Martins e Nogueira Jr. (2014) apresentam o jogo MUSIC-AR, que utiliza RA para auxiliar no ensino de propriedades sonoras como o timbre e a intensidade do som. Foram criadas duas aplicações, a primeira permite que a criança manipule objetos virtuais, ligados aos sons, e o segundo foca nos conceitos de intensidade do som, 
associando-o a animais virtuais. Também este trabalho se insere no contexto deste projeto, pois se propõe ensinar percepção musical.

Martins, Nunes e Correa (2015) apresentam um aplicativo para auxiliar no processo de ensino-aprendizagem musical. A aplicação contém nove módulos sobre os significados do som, da música e suas propriedades. Todos os módulos possuem um áudio, um texto explicativo e um vídeo demonstrativo. No módulo "As Propriedades do Som”, contém além destes elementos, uma aplicação de realidade aumentada. O projeto apresentado neste artigo deu origem a partir da continuação de Martins, Nunes e Correa (2015).

Os artigos encontrados na literatura mostram a relevância da área de RA para o ensino de música, principalmente com o público infantil. Este trabalho mostra a construção de um aplicativo musical de RA que vem complementar os estudos da literatura com a criação e disponibilização de um recurso educacional para apoio às aulas de musicalização infantil, ou também denominado percepção musical. O diferencial deste aplicativo em relação aos trabalhos encontrados na literatura é que possui propostas de atividades programadas, o Instrumenteca Quiz, que visa aumentar o aprendizado.

\section{Método}

Esta pesquisa foi desenvolvida em natureza exploratória de caráter qualitativo com intuito de obter um conhecimento sobre o tema. Inicialmente foi realizado um levantamento bibliográfico em livros e artigos sobre possíveis conteúdos que poderiam ser desenvolvidos nas aplicações. Em seguida, foi realizada a familiarização com os softwares de desenvolvimento e, paralelamente, a construção de duas aplicações, denominadas Instrumenteca RA e Instrumenteca Quiz. Esta pesquisa também tem caráter quantitativo, pois foi aplicado um questionário para 12 crianças, para pesquisa de opinião sobre sua satisfação, assim como a verificação do ganho de aprendizado.

\subsection{Descrição do Projeto}

A proposta do projeto foi desenvolver um objeto de aprendizagem composto por duas aplicações na área de Percepção Musical usando os softwares Unity e Vuforia, dirigidas aos alunos do ensino fundamental ou a usuários que gostam de música, trabalhando os conceitos básicos envolvidos em percepção musical e os tipos de instrumentos musicais. Todas as fases do processo de desenvolvimento das aplicações são detalhadas na próxima seção.

\subsection{Análise de Requisitos}

Primeiramente, foi realizada a técnica de brainstorming para reunir informações estimulando o pensamento criativo, aplicada à equipe de desenvolvimento. Nessa etapa foi decidido o tema do projeto, o software que seria usado e em qual plataforma seria desenvolvido. Em seguida, foram elencados os requisitos funcionais e não funcionais das aplicações. Foram considerados os seguintes requisitos funcionais:

- O sistema deverá reconhecer os marcadores referentes aos instrumentos musicais.

- O sistema deverá executar sons dos instrumentos musicais mediante a interação do usuário.

- O sistema deverá disponibilizar atividades de percepção musical.

Foram considerados requisitos não-funcionais: 
- O usuário deve ser capaz de navegar entre as telas das aplicações sem dificuldade.

- Ambiente físico: as aplicações executarão em smartphones Android.

- Interfaces: os resultados do Quiz estarão somente na aplicação do usuário, não haverá a possibilidade de compartilhar os resultados com outros usuários e nem em redes sociais.

- Quando o usuário estiver respondendo ao Quiz, não pode haver um delay entre as perguntas, o mesmo acontece quando é finalizado o Quiz. Portanto, não devem existir limitações quanto à velocidade de execução e/ou ao tempo de resposta e saída.

- O público-alvo é composto por crianças alfabetizadas na faixa etária entre $7 \mathrm{e}$ 14 anos, mas poderão haver usuários com outras idades que se interessarem por aprender ou aprimorar a percepção musical.

- Por questões de segurança e confidencialidade dos dados, o usuário não insere nenhuma informação pessoal nas aplicações.

- Em relação aos recursos, deve haver preocupação com a quantidade de armazenamento físico que será ocupada pelo sistema.

\subsection{Materiais}

As aplicações foram inicialmente desenvolvidas para dispositivos móveis Android, usando a plataforma de desenvolvimento Unity 5.6, linguagem de programação C\# para os scripts e Vuforia 6.2 para o desenvolvimento em RA para cardboard. Em relação aos componentes de hardware para o desenvolvimento, foi utilizado um computador HP Probook, com sistema operacional Windows 764 bits, processador Intel Core I5-4300M, 4GB de RAM, HD de 128GB de espaço.

O Instrumenteca está dividido em duas aplicações, Instrumenteca RA e Instrumenteca Quiz, descritas a seguir.

\subsubsection{Instrumenteca RA}

O Instrumenteca RA é uma aplicação de RA que proporciona ao usuário a possibilidade de visualizar um instrumento musical em 3D através de um celular acoplado em um Google Cardboard. Teve como principais objetivos: a) reconhecer visualmente os tipos de instrumentos musicais; b) reconhecer os sons dos instrumentos musicais; c) diferenciar os tipos de instrumentos musicais; d) ampliar o conhecimento de instrumentos musicais na parte visual e sonoro. Para tanto, a proposta inicial foi desenvolver um marcador para cada instrumento musical juntamente com o seu som. Posteriormente, foi montado um ambiente físico como um mural com os quatorze marcadores de cada instrumento.

O usuário veste o Google Cardboard e olha para um marcador individual para visualizar o instrumento em 3D e um fone de ouvido para ouvir o seu respectivo som - o uso de um cardboard e do fone de ouvido é para que o som esteja perto dos ouvidos do usuário e para dar maior sensação de imersão. A aplicação Instrumenteca RA é executada dentro do celular, que está encaixado no Google Cardboard (Figura 1). 

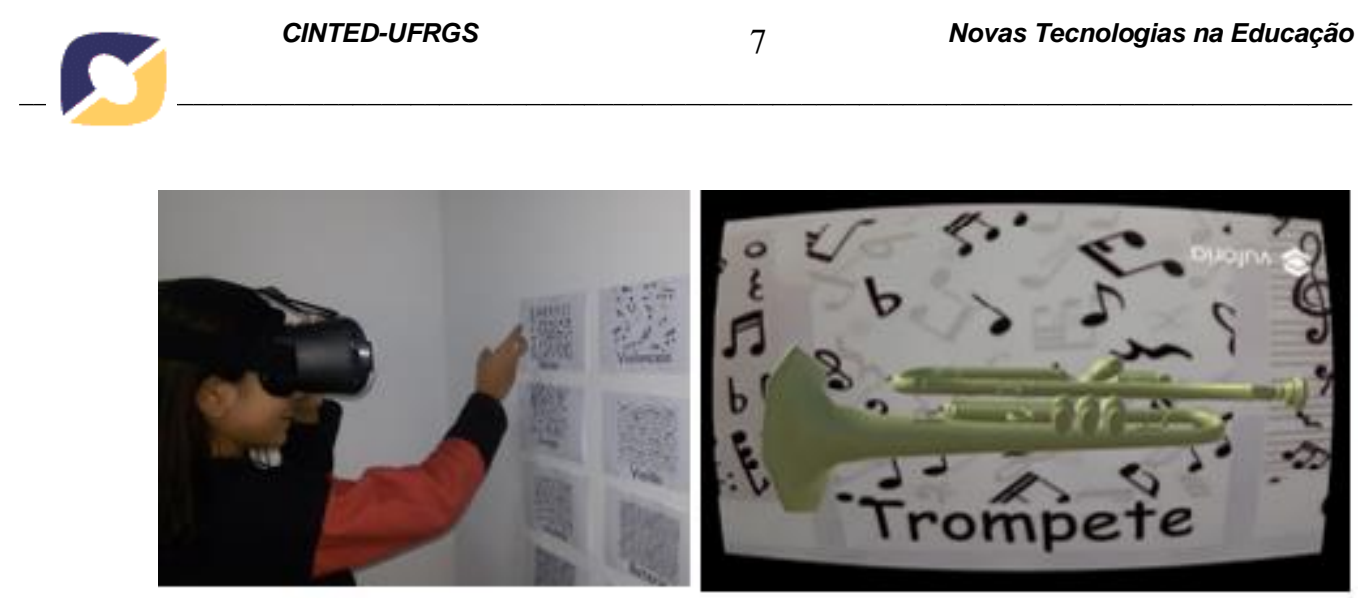

Figura 1. Google Cardboard para uso do Instrumenteca RA

\subsubsection{Instrumenteca Quiz}

Trata-se de uma aplicação Android (Figura 2), desenvolvida com Unity 3D, para testar os conhecimentos dos usuários sobre os conceitos sobre percepção musical e testar os conhecimentos vistos/aprendidos na primeira aplicação sobre os diferentes tipos de instrumentos musicais.

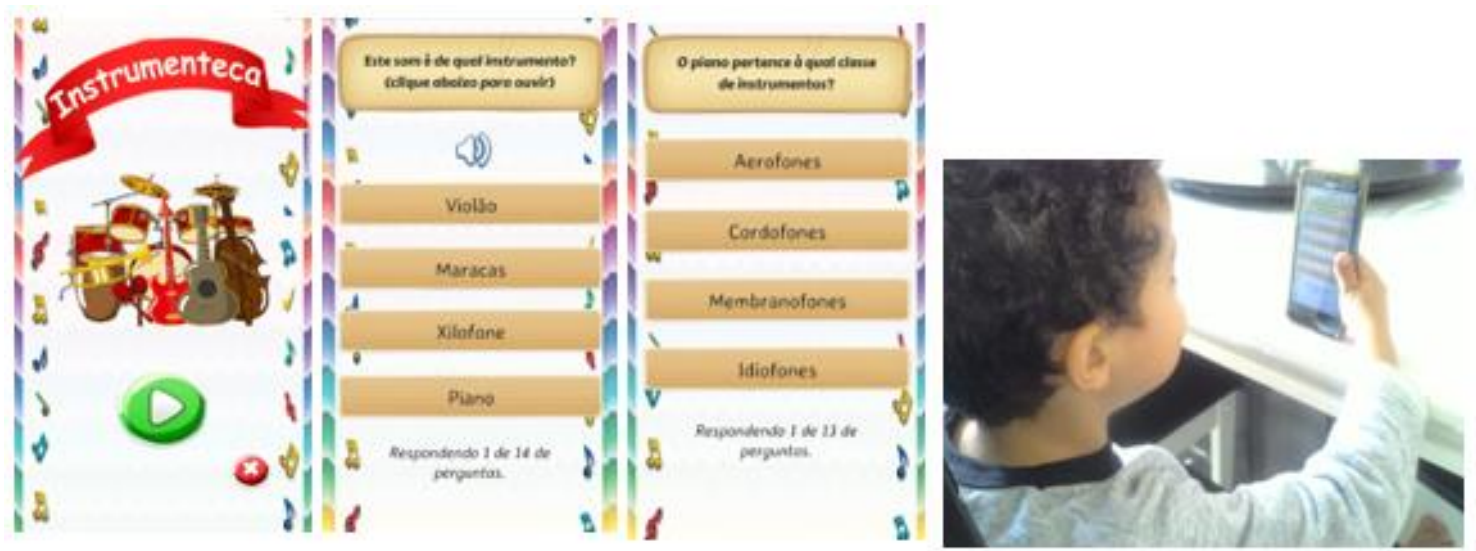

Figura 2. Telas do Instrumenteca Quiz

Durante a concepção do Instrumenteca Quiz foi criado um esboço do layout de cada tela da aplicação. Com as telas criadas, foram realizados testes verificando se os fluxos de dados estavam adequados, se o comportamento das aplicações estava consistente com as funções que deveriam ser executadas (exemplo: ao clicar em um botão, se ele realmente estava realizando a sua função de maneira correta) e se os objetivos das aplicações permanecem consistentes com os objetivos do sistema. Também foi verificado se a linguagem e os layouts estão de acordo com o público-alvo.

\subsection{Metodologia dos Testes com Usuários Finais}

A realização dos testes se deu em ambiente não controlado (no próprio domicílio da criança). As crianças escolhidas como público-alvo para os testes tiveram o aval dos pais. Para validar as aplicações, foi selecionado um grupo de 12 crianças de ambos os sexos, com idade variando entre seis e 14 anos. Um teste-piloto foi executado por seis adultos, para que fosse verificado se todos os passos que compunham o teste estavam corretos.

Para verificar o nível de conhecimento musical das crianças, foi realizado um préteste contendo três questões de múltipla escolha sobre o perfil do usuário e três questões relacionadas à percepção musical. As questões de perfil do usuário eram: gênero, idade, grau de escolaridade. Já as questões relacionadas à percepção musical se referiam ao nível 
de conhecimento musical, ao conhecimento das classes que os instrumentos pertenciam e ao uso de aplicações para o aprendizado de música.

Após o preenchimento do questionário de pré-teste, as crianças foram submetidas ao uso das aplicações. O tempo médio para a execução da aplicação foi de 20 minutos com o desvio padrão de dois minutos entre os testes. Após a utilização das aplicações, as crianças realizaram o pós-teste e, em seguida, responderam a um questionário de satisfação - que é uma das medidas de usabilidade, com questões propostas pelos autores. O questionário de pós-teste teve como objetivo medir o ganho de conhecimento de percepção musical e os diferentes tipos de instrumentos após utilizar as aplicações. Já o questionário de satisfação envolvia questões relacionadas à usabilidade da aplicação, tais como: quão importante é a utilização deste tipo de aplicação para o aprendizado; quão fácil é a utilização das aplicações; se a utilização das aplicações gerou algum tipo de desconforto visual; se a utilização das aplicações foi divertida.

\section{Resultados e Discussões}

Esta seção apresenta os resultados e as discussões sobre os testes realizados com as crianças com uso das aplicações Instrumenteca RA e Instrumenteca Quiz. A partir dos dados coletados no questionário de pré-teste, foi possível identificar que $100 \%$ das crianças nunca tiveram aula de música; $83 \%$ das crianças nunca utilizaram o Google Cardboard e, 92\% das crianças nunca utilizaram nenhum tipo de aplicação tecnológica para o aprendizado de música.

\subsection{Resultados do Aprendizado}

A Tabela 1 apresenta as respostas das crianças antes e após utilizar as aplicações. As perguntas tinham o mesmo conteúdo, em ordem aleatória.

Tabela 1. Quantidade de respostas corretas

\begin{tabular}{|c|c|c|}
\hline Sim & Não & \\
\hline $100 \%$ & $0 \%$ & \\
\hline \multicolumn{3}{|c|}{ O que você aprendeu utilizando a aplicação? } \\
\hline Diferenciar Instrumentos musicais & Objetos em 3D & Não aprendi nada \\
\hline $67 \%$ & $33 \%$ & $0 \%$ \\
\hline \multicolumn{3}{|c|}{$\begin{array}{l}\text { Resultado do Quiz: } \\
\text { questões respondidas corretamente }\end{array}$} \\
\hline Pré-teste & Pós-teste & Ganho no aprendizado \\
\hline 7,4 & 11,8 & $31 \%$ \\
\hline
\end{tabular}

Analisando a Tabela 1 é possível perceber que: antes da utilização das aplicações, as crianças acertaram, em média, 7,4 das questões, de um total de 14 questões, o que representa $53 \%$ de acerto. Após a utilização das aplicações, as crianças acertaram 11,8 questões das 14 questões, o que representa $84 \%$ de acerto. Isso corresponde a um ganho no aprendizado de $31 \%$ comparado ao seu desempenho na avaliação antes da utilização. Já no pós-teste, $100 \%$ das crianças declararam ter conhecido novos instrumentos durante a utilização das aplicações e a maioria das crianças, 67\%, declarou ter aprendido a diferenciar os instrumentos musicais após o uso das aplicações.

\subsection{Resultados dos Testes de Usabilidade}


Entre os valores respondidos pelas crianças a respeito da usabilidade das aplicações podemos destacar que:

- A maioria das crianças, $92 \%$, considerou muito importante a utilização de aplicações como estas para o aprendizado.

- $100 \%$ das crianças consideraram a utilização das aplicações muito fácil.

- $100 \%$ das crianças declararam que conseguem utilizar as aplicações sem a ajuda de um adulto.

- $100 \%$ das crianças declararam que as cores e desenhos das aplicações estavam adequadas.

- $100 \%$ das crianças não apresentaram desconfortos visuais, que poderiam ter sido causados pelo uso do Google Cardboard, durante a utilização das aplicações.

- $92 \%$ das crianças consideraram as aplicações muito divertidas.

- $100 \%$ das crianças recomendariam estas aplicações para um amigo.

- Não houve nenhum feedback negativo sobre a usabilidade das aplicações.

\section{Conclusão}

Este artigo apresentou o desenvolvimento de um objeto de aprendizagem em percepção musical, contendo duas aplicações, denominadas Instrumenteca RA e Instrumenteca Quiz, para apoiar o processo de aprendizagem musical, através da diferenciação dos instrumentos musicais. Os propósitos da aplicação são: possibilitar às crianças vivenciarem um amplo universo de elementos musicais e estimular a aprendizagem de percepção musical através da diferenciação de instrumentos musicais.

A avaliação de usabilidade (através da dimensão de satisfação), realizada com 12 crianças, mostrou que as aplicações são fáceis de utilizar, não sendo necessária a ajuda de um adulto para utilização. As crianças demonstraram muita satisfação e entusiasmo, dadas as características motivacionais da RA. Para todas as crianças participantes, foi o primeiro contato com um cardboard.

RA é uma ferramenta capaz de suportar diversos tipos de ambientes de aprendizagem em diferentes níveis de complexidade. No ensino musical, a RA permite a interação entre usuário e sistema de uma forma atrativa, eficiente e intuitiva. No caso das aplicações Instrumenteca RA e Instrumenteca Quiz, os conceitos básicos de percepção musical, como diferenciação dos instrumentos através de seu timbre, são reforçados fazendo com que as crianças consigam realizar os exercícios com sucesso.

Como perspectiva de continuidade deste estudo, em termos de implementação, pretende-se integrar novos instrumentos, pois atualmente são contemplados apenas 14 . Também pode-se incluir níveis de dificuldade para a diferenciação de instrumentos. Além disso, pode-se projetar o desenvolvimento de módulos com mais interatividade entre o objeto 3D e o usuário.

\section{REFERÊNCIAS}

Associação Brasileira de Educação Musical. ABEM. Disponível em $<$ http://abemeducacaomusical.com.br/artsg2.asp?id=20/>. Acesso em: 19 de março de 2016.

AZUMA, R. T. A survey of augmented reality. Presence: Teleoperators \& Virtual Environments, v. 6, n. 4, p. 355-385, 1997. 
BERNARDES, V. A percepção musical sob a ótica da linguagem. Revista da ABEM, v. 9, n. 6, 2014.

BERRY, R. et al. The augmented composer project: The music table. In: Proceedings of the 2nd IEEE/ACM International Symposium on Mixed and Augmented Reality. IEEE Computer Society, 2003. p. 338.

BILLINGHURST, M. Augmented reality in education. New horizons for learning, v. 12, n. 5, 2002.

DE ARAUJO BORGES, S.; PENNA, M. O estudo da percepção musical em um curso técnico em instrumento musical: um projeto de pesquisa. In: XXII Congresso Nacional da Associação Brasileira de Educação Musical. 2015.

BOWER, M. et al. Augmented Reality in education-cases, places and potentials. Educational Media International, v. 51, n. 1, p. 1-15, 2014.

CORRÊA, A. G. D.; FICHEMAN, I. K.; DE DEUS LOPES, R. Interface Musical Tangível Desenvolvida com Tecnologia de Realidade Aumentada. Revista de Exatas e TECnológicas, v. 3, n. 1, p. 07-17, 2012.

CORRÊA, A. G. D. et al. Desenvolvimento de um Livro Interativo em Realidade Aumentada para Ensino e Aprendizagem Musical. Renote, v. 10, n. 2, 2013.

GOMEZ, L.; MARTINS, V. F.; NOGUEIRA JR., J. EDUCAÇÃO MUSICAL: O SOFTWARE MUSIC-AR PARA O ENSINO DE PERCEPÇÃO SONORA PARA CRIANÇAS DA PRÉ-ESCOLA. In: Proceedings of World Congress on Communication and Arts. 2014. p. 255-259.

GORDON, E. E. Teoria de aprendizagem musical: competências, conteúdos e padrões (Maria de Fátima Albuquerque, trad.). Lisboa, PT: Fundação Calouste Gulbenkian, 2000.

JESUS, E. A.; URIARTE, M. Z.; RAABE, A. L. A. Zorelha: Um objeto de aprendizagem para auxiliar o Desenvolvimento da percepção musical em crianças de 4 a 6 anos. Brazilian Journal of Computers in Education, v. 18, n. 01, p. 91, 2010.

JOHNSON, L. et al. NMC horizon report: 2016 higher education edition. The New Media Consortium, 2016.

KIRNER, C.; KIRNER, T. G. Virtual reality and augmented reality applied to simulation visualization. In: Simulation and Modeling: Current Technologies and Applications. IGI Global, 2008. p. 391-419.

NUNES, S. S.; MARTINS, V. F.; CORREAA, A. G. D. Open educational resources to support musical education. In: Information Systems and Technologies (CISTI), 2015 10th Iberian Conference on. IEEE, 2015. p. 1-6.

RODRIGUES, C. A. M.; ROSIN, S. M. A importância do ensino de música para o desenvolvimento infantil. Maringá: UEMA, 2011.

RODRIGUES, J. N. A música na educação infantil: um recurso pedagógico que favorece o desenvolvimento integral das crianças. 2016. Trabalho de Conclusão de Curso. Universidade Federal do Rio Grande do Norte.

SILVA, E. M. P.; TONI, F. C. Instrumentos Musicais da Coleção Mário de Andrade. Revista do Instituto de Estudos Brasileiros, n. 31, p. 197-206, 1990.

SOUZA, J. Educação musical e práticas sociais. Revista da ABEM, v. 12, n. 10, 2014. 